\title{
Protonation effect on the excited state behaviour of some aza-analogues of $E E$-distyrylbenzene
}

\author{
Gabriella Ginocchietti, Ugo Mazzucato, ${ }^{\dagger}$ and Anna Spalletti \\ Dipartimento di Chimica, Università di Perugia, I-06123 Perugia, Italy
}

\begin{abstract}
The photobehaviour of four aza-analogues of EE-distyrylbenzene bearing the heteroatom in the two side rings [1,4-di-(2-pyridylethenyl)benzene] or in the central ring [2,5-di-(phenylethenyl)pyridine, 2,6-di(phenylethenyl)pyridine and the corresponding diene, 2,6-di(phenylbutadienyl)pyridine] has been investigated in aqueous solutions at $\mathrm{pH} 2$ and 9, where the molecules are in mono-protonated and neutral forms, respectively. The type of conjugation, linear for the first two compounds and crossed for the others, is particularly important in determining the spectral and photochemical properties. The main effects of the protic solvent are a reduction of the radiative deactivation, prevalent in the corresponding hydrocarbons, and an increase of the reactive deactivation, very modest in the hydrocarbons. The increase in photoreactivity to detriment of fluorescence, observed in protic medium with respect to non-polar solvents, is drastically emphasized in acid medium where the fluorescence quantum yield decreases by an order of magnitude leading to high values of the $\mathrm{EE} \rightarrow \mathrm{ZE}$ photoisomerization quantum yield. The presence of conformational isomerism (two main conformers were detected) has been observed in both basic and acid media for compounds with crossed conjugation and for the linearly conjugated 2,5 substituted pyridine. The results obtained give a picture of the different photobehaviour of the neutral and protonated species and indicate the experimental conditions to favour a specific relaxation pathway.
\end{abstract}

\section{INTRODUCTION}

The excited state behaviour of aza-analogues of stilbenoid compounds, where one or two phenyl groups are replaced by pyridyl groups, has been extensively investigated in our [1-8] and other [913] laboratories. The fluorescence properties and the photoisomerization mechanism have been interpreted in terms of the competition among the radiative, non-radiative and reactive relaxation processes. The acid-base equilibria and the protonation effect on the photobehaviour in aqueous solutions have been studied in this laboratory for $n$ styrylpyridines [14-16], naphthyl- and phenanthryl-npyridylethenes [17, 18], n,n'-dipyridylethenes [19] and 1,4-di-(n-pyridyl)butadienes (n,n' $=2,3$ and 4) [20].

This paper describes the acid-base equilibria and the protonation effect on the photobehaviour of some aza-analogues of distyrylbenzene, bearing the nitrogen heteroatom in the two side aryl groups or in the central aromatic ring, previously studied in organic solvents [21-23]. The main aim was to compare their excited state properties with those of the corresponding hydrocarbons, 1,4-distyrylbenzene [1,4$(\mathrm{PhE})_{2} \mathrm{~B}$, linear conjugation] and 1,3-distyrylbenzene [1,3-(PhE $)_{2} \mathrm{~B}$, crossed conjugation] and the corresponding dienes [21-24]. Replacement of benzene rings with pyridine rings or protonated pyridinium rings in stilbenoid compounds can differently affect the photobehaviour. Generally, the heteroatom has been found to reduce the torsional barrier to

\footnotetext{
†E-mail: mazzucat@unipg.it
}

isomerization thus leading to an increase of the photoreaction rate $[7,25,26]$. However, in some cases, the presence of $n, \pi^{*}$ states introduced by the nitrogen atom have been found to favour internal conversion (IC), thus reducing both fluorescence and photoreaction. In the latter case, protonation of the pyridyl group is expected to avoid the deactivating effect of the $n$, $\pi^{*}$ states, thus potentially increasing the contribution of the radiative and/or reactive relaxation pathways [16-18].

The results now obtained in aqueous solutions, at basic and acid pH's, are compared to those previously obtained for the corresponding hydrocarbons and for the same aza-analogues in organic solvents. They show that changes in $\mathrm{pH}$ may favour a selective relaxation pathways of $S_{1}$; this can be important since potential applications of these compounds in material science may require, depending on their nature, a predominance of the radiative pathway or the reactive one [24].

\section{EXPERIMENTAL}

The four EE compounds investigated (Scheme 1) bear the heteroatom in the two side rings $[1,4-$ di-(2-pyridylethenyl)benzene (1)] or in the central ring [2,5-di-(phenylethenyl)pyridine (2), 2,6-di(phenylethenyl)pyridine (3) and 2,6-di(phenylbutadienyl)pyridine (4). Conjugation of compounds 1 and 2 is linear while crossed conjugation characterizes compounds 3 and 4 [24]. They were prepared by the Wittig reaction at the Padua laboratory for previous works carried out in organic solvents [21-23]. 


\section{LINEAR CONJUGATION}

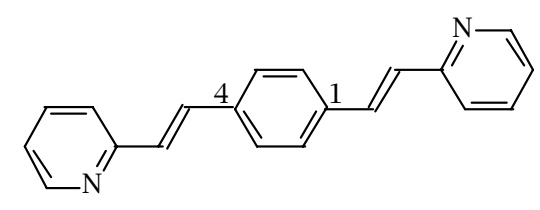

1,4-di-(2-pyridylethenyl)benzene (1)

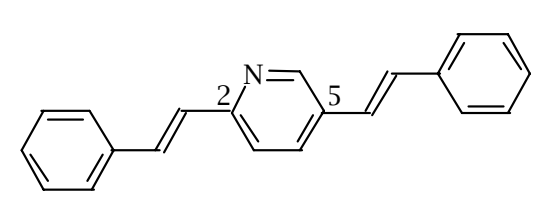

2,5-di-(phenylethenyl)pyridine (2)

\section{CROSSED CONJUGATION}<smiles>C(=C/c1cccc(/C=C/c2ccccc2)n1)\c1ccccc1</smiles>

2,6-di-(phenylethenyl)pyridine (3)<smiles>C(/C=C/c1cccc(/C=C/C=C/c2ccccc2)n1)=C\c1ccccc1</smiles>

2,6-di-(phenylbutadienyl)pyridine (4)

Scheme 1.

In the present work, a 30/70 (v/v) $\mathrm{CH}_{3} \mathrm{CN} / \mathrm{H}_{2} \mathrm{O}$ mixture was generally used, the percentage of organic solvent, which reached $40 \%$ for compound 4 , being required by the scarce solubility in water.

The absorption measurements were carried out by a Perkin-Elmer Lambda 800 spectrophotometer. The fluorescence spectra were measured by a Spex Fluorolog-2 F112AI spectrofluorimeter. Dilute solutions (absorbance $<0.1$ at the excitation wavelength, $\lambda_{\text {exc }}$ ) were used for the fluorimetric measurements. Except when otherwise specified, the emission and photoreaction quantum yields were determined at $\lambda_{\text {exc }}$ corresponding to the maximum of the first absorption band $\left(\lambda_{\max }\right) .9$,10-diphenylanthracene was used as fluorimetric standard $\left(\phi_{\mathrm{F}}=0.90\right.$ in deareated cyclohexane [27]). For photochemical measurements (potassium ferrioxalate in water as actinometer), a $150 \mathrm{~W}$ high pressure xenon lamp coupled with a monochromator was used. The photoreaction (solute concentrations $\sim 10^{-4} \mathrm{M}$ ) was monitored by HPLC. A Waters apparatus equipped with analytical Simmetry C18 $(4.6 \times 250 \mathrm{~mm}$; $5 \mu \mathrm{m})$ or Prontosil 200-3-C30 $(4.6 \times 250 \mathrm{~mm} ; 3 \mu \mathrm{m})$ columns and UV detector was used. Fluorescence lifetimes were measured by an Edinburgh Instrument $199 \mathrm{~S}$ spectrofluorimeter, using the single photon counting method, or by a Spex Fluorolog- 2 apparatus, based on the phase modulation technique, described elsewhere [26]. The fluorimetric and photochemical mea- surements were carried out in solutions de-areated by bubbling nitrogen. All parameters reported in the tables are averages of at least three independent experiments with mean deviations of ca. $10 \%$ for fluorescence and photoisomerization quantum yields and ca. $15 \%$ for the fluorescence lifetimes.

The $\mathrm{pK}_{\mathrm{a}}$ values were obtained by spectrophotometric titration, measuring the $\mathrm{pH}$ values by a Sargent PXB pH-meter with glass electrode. Britton buffers at constant ionic strength (0.01) were used in the 2-12 pH range, and $\mathrm{HClO}_{4}$ and $\mathrm{NaOH}$ solutions for more acid and alkaline pH's, respectively. For the second protonation of the bibasic compound $\mathbf{1}$, concentrated $\mathrm{H}_{2} \mathrm{SO}_{4}$ solutions were used, their acidity being evaluated by the $\mathrm{H}_{0}$ acidity function. For the weaker base 2 , the small percentage of neutral species still present at $\mathrm{pH} 2$ was found (check at $\mathrm{pH} 1$ ) to have negligible effect on the radiative and reactive parameters.

Theoretical calculations on the energy levels, electronic structure and transition probabilities were carried out by the ZINDO/S method using Hyperchem (version 6) software and PM3 optimized geometries. The calculations of configuration interaction included 81 $(9 \times 9)$ single excited configurations. The overlap weighting factor was set $\mathrm{f}_{\mathrm{p} \pi}=0.585$ for the neutral and biprotonated molecules and $\mathrm{f}_{\mathrm{p} \pi}=0.70$ for the monoprotonated species to obtain a good accord with the experimental electronic spectra. 


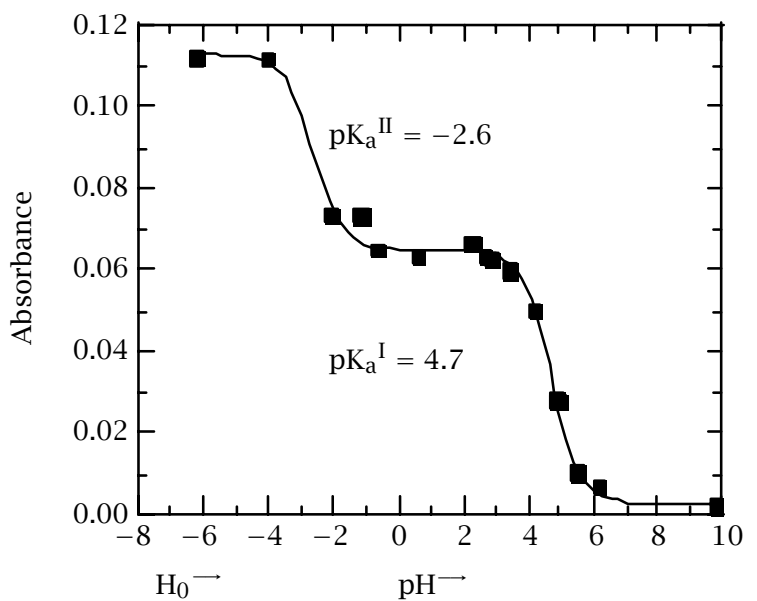

Figure 1. Spectrophotometric titration curve of 1 in 30/70 $v / v \mathrm{CH}_{3} \mathrm{CN} / \mathrm{H}_{2} \mathrm{O}$.

\section{RESULTS AND DISCUSSION}

3.1. Acid-base equilibria. This reaserch work started with the measurements of the acid-base properties of the investigated compounds in order to choose the $\mathrm{pH}$ values suitable to study the photobehaviour of the neutral and protonated species. The equilibrium constants $\left(\mathrm{pK}_{\mathrm{a}}\right.$, where $\mathrm{K}_{\mathrm{a}}$ is the dissociation constant of the conjugated acid) of some aza-analogues of $E E$ distyrylbenzene were measured by spectrophotometric titration. Figure 1 shows, as an example, the titration curve of the bibasic compound $\mathbf{1}$. Table 1 collects the ground state $\mathrm{pK}_{\mathrm{a}}$ 's (not far from the known value of pyridine, ca. 5.2 [27]) together with the corresponding values for the first excited singlet state $\left(\mathrm{pK}_{\mathrm{a}}{ }^{*}\right)$ calculated by the Förster cycle. The high $\mathrm{pK}_{\mathrm{a}}{ }^{*}$ values (> 8) indicate that these molecules become much stronger bases on excitation, as found for the aza-analogues of several diaryl-ethenes [14-19] and the corresponding dienes [20].

Since these compounds exist in solution as a mixture of different conformers in equilibrium (see below), the spectrometric titration gives $\mathrm{pK}_{\mathrm{a}}$ values which can be considered averaged values for the conformer mixtures. However, one can reasonably expect that different conformers have practically the same acid-base properties.

On the basis of these $\mathrm{pK}_{\mathrm{a}}$ values, the effect of protonation on the spectral properties and on the photophysical and photochemical behaviour was investigated at $\mathrm{pH}$ values of 2 and 9, corresponding to the protonated and neutral species, respectively. For the bibasic compound 1, pH 2 corresponds to the monoprotonated species; the biprotonated species was investigated at $\mathrm{H}_{0}=-4.9$.

\subsection{Spectral properties and conformational equi-} libria. Figures 2 and 3 show the absorption and fluo-
Table 1. $\mathrm{pK}_{\mathrm{a}}$ and $\mathrm{pK}_{\mathrm{a}}{ }^{*}$ for the investigated compounds in $30 / 70 \mathrm{CH}_{3} \mathrm{CN} / \mathrm{H}_{2} \mathrm{O}$ at room temperature, measured by spectrophotometric titration and calculated by the Förster cycle.

\begin{tabular}{ccc}
\hline compound & $\mathrm{pK}_{\mathrm{a}}$ & $\mathrm{pK}_{\mathrm{a}}{ }^{*}$ (calc.) \\
\hline 1 & 4.7 & 9.2 \\
2 & 3.8 & 8.5 \\
3 & 4.3 & 10.1 \\
$4^{\mathrm{a})}$ & 4.2 & 10.8 \\
\hline
\end{tabular}

a) In $40 / 60 \mathrm{CH}_{3} \mathrm{CN} / \mathrm{H}_{2} \mathrm{O}$.
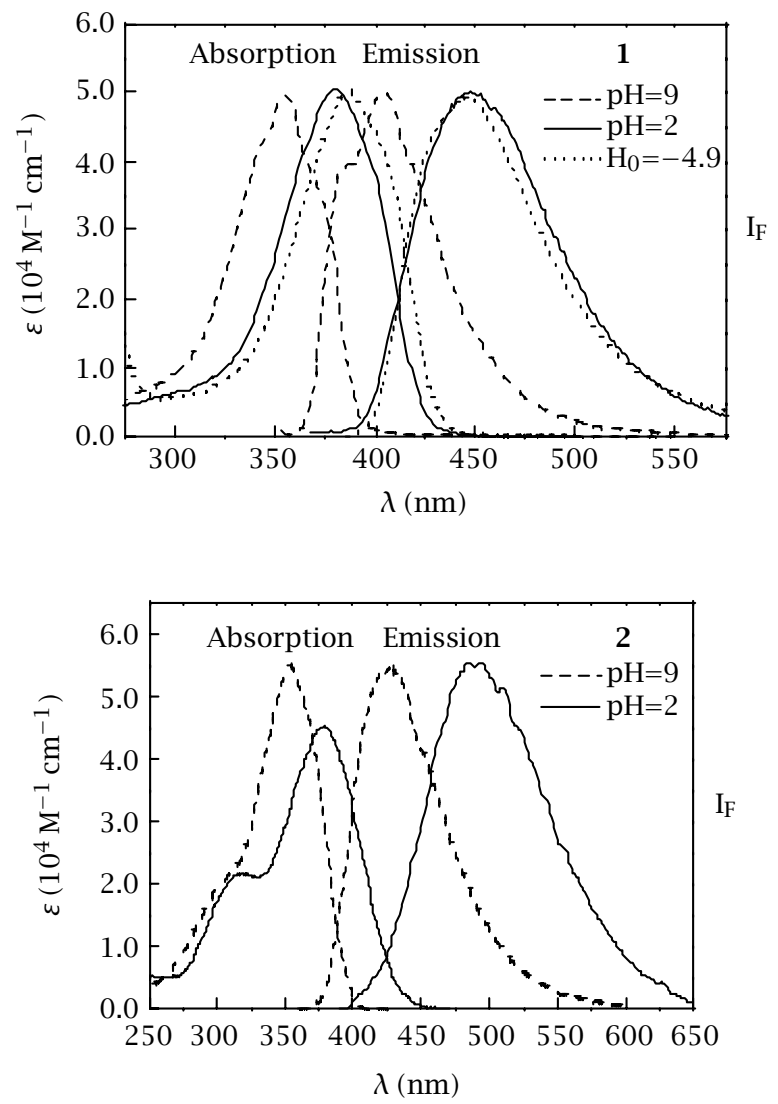

Figure 2. Spectra of neutral, mono- and bi-protonated 1 and neutral and protonated 2 in $30 / 70 v / v \mathrm{CH}_{3} \mathrm{CN} / \mathrm{H}_{2} \mathrm{O}$.

rescence spectra of the neutral and protonated species of the four aza-compounds investigated. Protonation induces a marked red-shift of the spectra with respect to those of the neutral species. For the two compounds with crossed conjugation (3 and 4), an inversion of the intensity of the two first absorption bands was found on going from basic to acid medium.

This behaviour can be related to the shift of the conformational equilibrium towards the more elongated rotamer (see below).

MO calculations of the energy levels, transition probabilities and formation enthalpies of the neutral and protonated forms of these aza-compounds 

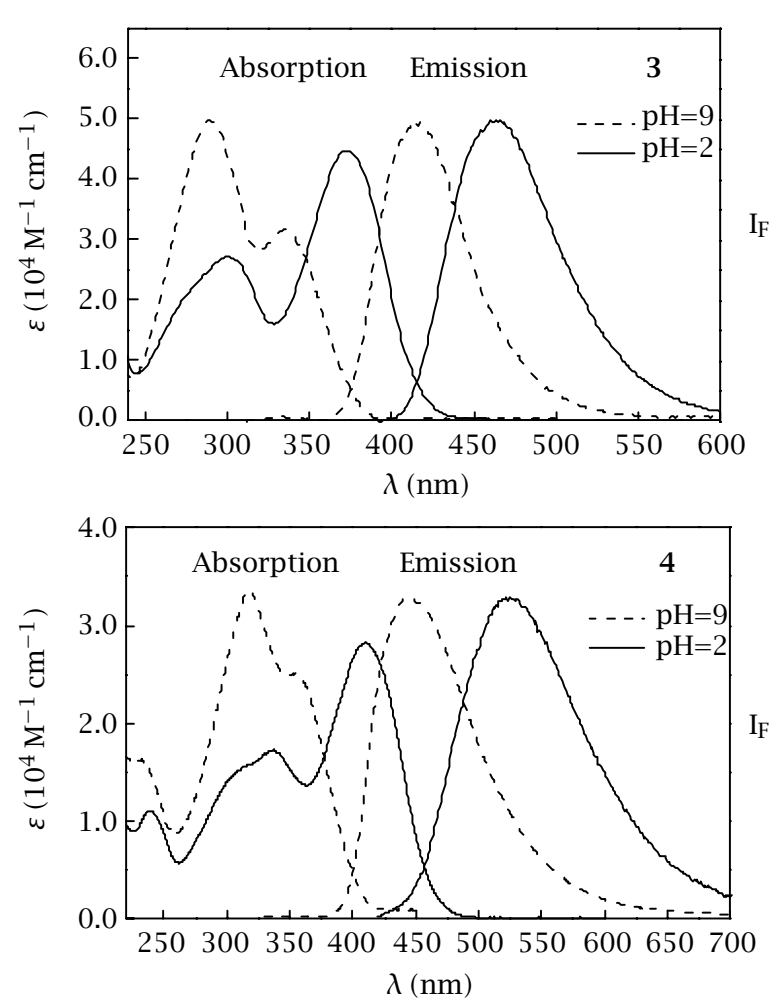

Figure 3. Spectra of neutral and protonated 3 in $30 / 70 \mathrm{v} / \mathrm{v}$ $\mathrm{CH}_{3} \mathrm{CN} / \mathrm{H}_{2} \mathrm{O}$ and 4 in $40 / 60 v / v \mathrm{CH}_{3} \mathrm{CN} / \mathrm{H}_{2} \mathrm{O}$.

(Tables 2 and 3) led to results in good agreement with the experimental spectra. The computed spectra well reproduce the large red-shift (over than $30 \mathrm{~nm}$ ) observed after protonation. Those for the second protonation of 1,4-(2PE $)_{2} \mathrm{~B}$ remained practically unchanged; only a further small red shift was found in the experimental spectra.

The computed enthalpy difference of the conformers of the neutral species is small (in the range 0.2$0.8 \mathrm{kcal} / \mathrm{mol}$ ) suggesting the occurrence of a dynamic equilibrium among different conformers in fluid solutions at room temperature. The observed photobehaviour of the compounds with pyridine as central ring clearly confirmed the presence of at least two different conformers [21, 22].

Indeed, as reported for the hydrocarbons [21, 23, $24]$, the very small effects of the excitation wavelength ( $\left.\lambda_{\text {exc }}\right)$ on the fluorescence spectra of the 1,4-substituted compound 1, as well as its mono-exponential decay, made the detection of the expected rotamers rather difficult (probably because of very similar spectral and photophysical properties of the existing conformers). A clearer wavelength dependence and a poly-exponential decay were found for the other compounds, particularly for 2 and 3 (see below). Figure 4 shows the wavelength effect on the spectra of compound 2, as an example.
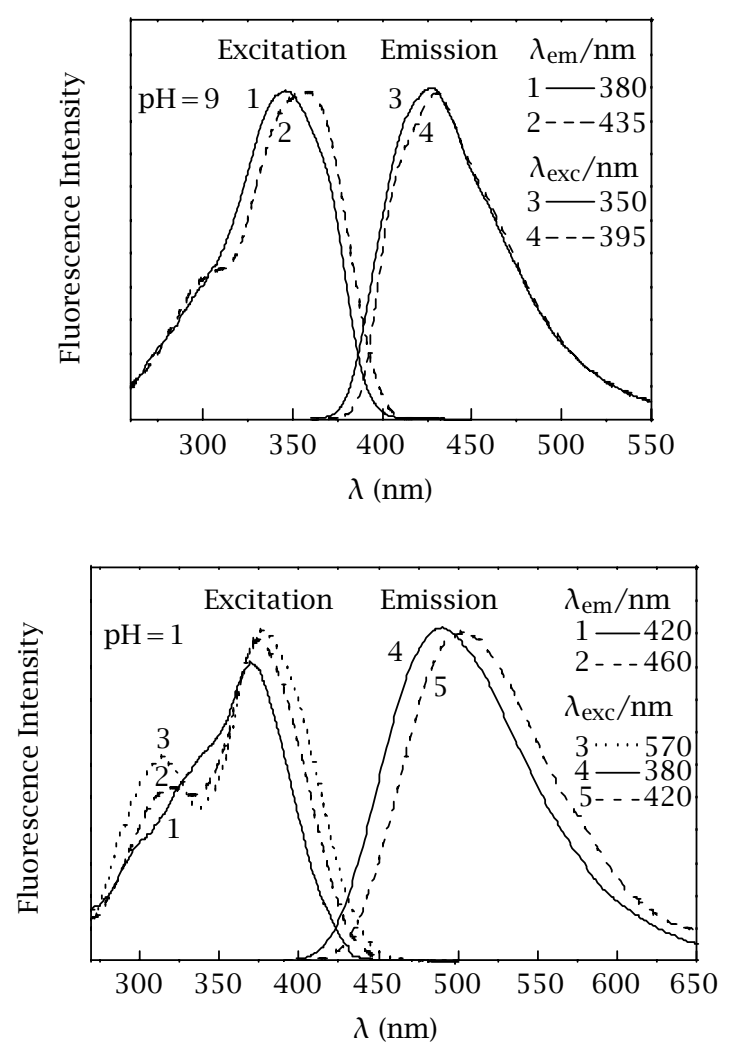

Figure 4. Effect of $\lambda_{\mathrm{exc}}$ and $\lambda_{\mathrm{em}}$ on the emission and excitation spectra of compound 2 in $30 / 70 v / v \mathrm{CH}_{3} \mathrm{CN} / \mathrm{H}_{2} \mathrm{O}$ at different pHs.

The results of MO calculations in Tables 2 and 3 refer to the two most different conformers. For the symmetric compound $\mathbf{1}$, rotation of the pyridylvinyl group leads to species with practically the same heats of formation and spectral properties, whereas small, but more significant, differences were found by rotation of the side pyridyl groups, which changes the distance of the heteroatom with respect to the ethenic hydrogens. The two conformers reported in Table 2 are then the more elongated ones, with different positions of the heteroatom. On the other hand, for compounds 2, 3 and 4, the two most different configurations are those obtained by rotation of the two styryl or arylbutadienyl groups. For compound 2, data in Table 2 refer to elongated species whereas, for the cross-conjugated compounds 3 and 4, the most different conformations (I and II in Table 3) are the most elongated and constricted ones.

For compounds 2, 3 and 4, spectral changes were also found at $\mathrm{pH} 2$ where a larger enthalpy difference favours the less constricted conformer I which absorbs at shorter wavelengths. This allowed the quasi-pure spectrum of the bathochromic rotamer II to be obtained by selective excitation at the tail of the absorption spectrum (Figure 4). 
Table 2. MO calculations of electronic spectra (transition energy, $\lambda$, and oscillator strength, $\boldsymbol{f}$ ) and heats of formation $\left(\Delta \mathbf{H}_{\mathrm{f}}^{\circ}\right)$ for the two most different conformations of neutral and protonated compounds $\mathbf{1}$ and $\mathbf{2}$ (linear conjugation).

\begin{tabular}{|c|c|c|c|c|c|}
\hline molecule & rotamer & $\Delta \mathrm{H}_{\mathrm{f}}^{\circ}(\mathrm{kcal} / \mathrm{mol})$ & $\lambda(\mathrm{nm})$ & $\mathrm{f}$ & \\
\hline \multirow{6}{*}{1} & & 112.66 & 348.5 & 2.00 & $\mathrm{~S}_{1}$ \\
\hline & & & 296 & 0.004 & $\mathrm{~S}_{2}$ \\
\hline & I & & 278 & 0.058 & $\mathrm{~S}_{4}$ \\
\hline & & 113.16 & 348 & 1.96 & $\mathrm{~S}_{1}$ \\
\hline & & & 296 & 0.003 & $\mathrm{~S}_{3}$ \\
\hline & II & & 279 & 0.15 & $\mathrm{~S}_{4}$ \\
\hline \multirow{7}{*}{$1-\mathrm{H}^{+}$} & & 259.97 & 422 & 1.76 & $\mathrm{~S}_{1}$ \\
\hline & & & 290 & 0.17 & $\mathrm{~S}_{2}$ \\
\hline & & & 281 & 0.21 & $\mathrm{~S}_{3}$ \\
\hline & $\mathrm{I}$ & & & & \\
\hline & & 261.44 & 426 & 1.67 & $\mathrm{~S}_{1}$ \\
\hline & & & 290 & 0.17 & $\mathrm{~S}_{2}$ \\
\hline & II & & 281 & 0.36 & $\mathrm{~S}_{3}$ \\
\hline \multirow{7}{*}{$1-\mathrm{H}_{2}^{++}$} & & 444.95 & 421 & 1.95 & $\mathrm{~S}_{1}$ \\
\hline & & & 307 & 0.018 & $\mathrm{~S}_{3}$ \\
\hline & & & 280 & 0.13 & $\mathrm{~S}_{4}$ \\
\hline & I & & & & \\
\hline & & 448.02 & 422 & 1.86 & $\mathrm{~S}_{1}$ \\
\hline & & & 307 & 0.012 & $\mathrm{~S}_{3}$ \\
\hline & II & & 277 & 0.275 & $\mathrm{~S}_{4}$ \\
\hline \multirow{6}{*}{2} & & 105.45 & 350 & 1.86 & $\mathrm{~S}_{1}$ \\
\hline & & & 303 & 0.13 & $\mathrm{~S}_{2}$ \\
\hline & I & & & & \\
\hline & & 105.69 & 353 & 1.70 & $\mathrm{~S}_{1}$ \\
\hline & & & 297 & 0.37 & $\mathrm{~S}_{2}$ \\
\hline & II & & 272 & 0.026 & $\mathrm{~S}_{3}$ \\
\hline \multirow{6}{*}{$2-\mathrm{H}^{+}$} & & 250.29 & 384 & 1.45 & $\mathrm{~S}_{1}$ \\
\hline & & & 312 & 0.89 & $\mathrm{~S}_{2}$ \\
\hline & 1 & & & & \\
\hline & & 251.58 & 395 & 1.24 & $\mathrm{~S}_{1}$ \\
\hline & & & 303 & 0.953 & $\mathrm{~S}_{2}$ \\
\hline & II & & 295 & 0.307 & $\mathrm{~S}_{3}$ \\
\hline
\end{tabular}

The first transition, characterized by a high value of the oscillator strength, populates an allowed excited singlet state for all compounds investigated. The splitting of the first absorption band into two components, characteristic of aza-compounds with the ethenic bridge in ortho position with respect to the heteroatom [30], is not much evident when the pyridine is the side group, as in compound 1. A weak hypsochromic band (almost 10 times less intense than the bathochromic one) is observable only for conformer II, having the $\mathrm{N}$ atom closer to the ethenic hydrogen, and for the protonated species. A slightly more evident behaviour, particularly at $\mathrm{pH} 2$, was found for compound
2, with the $\mathrm{N}$ atom in the central ring and with linear conjugation. The splitting becomes more important in the cross-conjugated compounds 3 and 4 . The calculations on the neutral and protonated, more constricted, species of these compounds (rotamers II in Table 3) gave the first transition less intense than that of the hypsochromic band in the absorption spectra. This is in nice agreement with the spectra of Figure 2 showing an inversion in the intensity of the first two absorption bands in the protonated species with respect to those of neutral ones, pointing to a prevalence of the hypsochromic (more elongated) conformer I in acid medium. Indeed, the calculated oscillator strength of 
Table 3. MO calculations of electronic spectra (transition energy, $\lambda$, and oscillator strength, $\boldsymbol{f}$ ) and heats of formation $\left(\Delta \mathbf{H}_{\mathbf{f}}^{\circ}\right)$ for the most two different conformations of neutral and protonated compounds 3 and 4 (crossed conjugation).

\begin{tabular}{|c|c|c|c|c|c|}
\hline molecule & rotamer & $\Delta \mathrm{H}_{\mathrm{f}}^{\circ}(\mathrm{kcal} / \mathrm{mol})$ & $\lambda(\mathrm{nm})$ & $\mathrm{f}$ & \\
\hline \multirow{8}{*}{3} & \multirow[b]{3}{*}{ I } & \multirow[t]{3}{*}{106.05} & 337 & 1.25 & $\mathrm{~S}_{1}$ \\
\hline & & & 296 & 0.32 & $\mathrm{~S}_{2}$ \\
\hline & & & 276 & 0.902 & $\mathrm{~S}_{6}$ \\
\hline & \multirow[b]{5}{*}{ II } & \multirow[t]{5}{*}{106.58} & 343 & 0.75 & $\mathrm{~S}_{1}$ \\
\hline & & & 288 & 0.019 & $\mathrm{~S}_{2}$ \\
\hline & & & 287 & 1.07 & $\mathrm{~S}_{3}$ \\
\hline & & & 282 & 0.072 & $\mathrm{~S}_{5}$ \\
\hline & & & 275 & 0.47 & $\mathrm{~S}_{6}$ \\
\hline \multirow{7}{*}{$3-\mathrm{H}^{+}$} & \multirow[b]{3}{*}{ I } & \multirow[t]{3}{*}{247.77} & 388 & 1.80 & $\mathrm{~S}_{1}$ \\
\hline & & & 293 & 0.373 & $\mathrm{~S}_{2}$ \\
\hline & & & 268 & 0.033 & $\mathrm{~S}_{3}$ \\
\hline & & \multirow[t]{4}{*}{250.67} & 396 & 1.085 & $\mathrm{~S}_{1}$ \\
\hline & & & 291 & 1.046 & $\mathrm{~S}_{2}$ \\
\hline & & & 267 & 0.41 & $\mathrm{~S}_{3}$ \\
\hline & II & & & & \\
\hline \multirow{6}{*}{4} & \multirow[b]{3}{*}{ I } & \multirow[t]{3}{*}{133.86} & 354 & 2.28 & $\mathrm{~S}_{1}$ \\
\hline & & & 315 & 0.19 & $\mathrm{~S}_{2}$ \\
\hline & & & 293 & 0.79 & $\mathrm{~S}_{3}$ \\
\hline & \multirow{3}{*}{ II } & \multirow[t]{3}{*}{134.72} & 363 & 0.834 & $\mathrm{~S}_{1}$ \\
\hline & & & 307 & 2.20 & $\mathrm{~S}_{2}$ \\
\hline & & & 293 & 0.24 & $\mathrm{~S}_{3}$ \\
\hline \multirow{9}{*}{$4-\mathrm{H}^{+}$} & \multirow[b]{4}{*}{ I } & \multirow{4}{*}{274.00} & 434 & 2.44 & $\mathrm{~S}_{1}$ \\
\hline & & & 330 & 0.25 & $\mathrm{~S}_{2}$ \\
\hline & & & 294 & 0.054 & $\mathrm{~S}_{3}$ \\
\hline & & & 275 & 0.48 & $\mathrm{~S}_{4}$ \\
\hline & \multirow[b]{5}{*}{ II } & \multirow[t]{5}{*}{277.33} & 446 & 1.03 & $\mathrm{~S}_{1}$ \\
\hline & & & 328 & 1.67 & $\mathrm{~S}_{2}$ \\
\hline & & & 295 & 0.843 & $\mathrm{~S}_{3}$ \\
\hline & & & 274 & 0.114 & $\mathrm{~S}_{4}$ \\
\hline & & & & & \\
\hline
\end{tabular}

the first two absorption transitions of the protonated 3 compound is very similar for the more constricted rotamer II, but higher for the first transition in the case of rotamer I. A similar behaviour was found for compound 4 . However, it has to be noted that, for the latter, more complicate conformational equilibria exist in solutions since rotation around the quasi-single bonds of the olefin chain can come into play.

The calculated heat of formation for the crossconjugated compounds points to the elongated species as the most stable one, but the results on the relative stability, in the case of the neutral species, could be 
Table 4. Effect of the nitrogen heteroatom on the photobehaviour of 1,4-(2PE) ${ }_{2} \mathrm{~B}(\mathbf{1}$, with linear conjugation) in non-polar solvent and in $30 / 70 \mathrm{CH}_{3} \mathrm{CN} / \mathrm{H}_{2} \mathrm{O}$ at $\mathrm{pH} 9$ and 2 and $\mathrm{H}_{0}=-4.9$.

\begin{tabular}{cccccc}
\hline $\begin{array}{c}\text { solvent } \\
\text { compound }\end{array}$ & $1,4-(\mathrm{PhE})_{2} \mathrm{~B}^{\mathrm{a})}$ & $1^{\mathrm{a})}$ & 1 & \multicolumn{2}{c}{ aqueous medium } \\
\hline$\Phi_{\mathrm{F}}$ & 0.84 & 0.28 & 0.13 & 0.018 & 0.033 \\
$\tau_{\mathrm{F}}(\mathrm{ns})$ & 1.7 & 0.56 & 0.35 & 0.12 & 0.27 \\
$\mathrm{k}_{\mathrm{F}}\left(10^{8} \mathrm{~s}^{-1}\right)$ & 4.3 & 5.0 & 3.7 & 1.5 & 1.2 \\
$\Phi_{\mathrm{EE} \rightarrow \mathrm{ZE}}$ & $<0.001$ & 0.25 & 0.31 & 0.56 & 0.64 \\
\hline
\end{tabular}

a) From ref. [22].

Table 5. Effect of the nitrogen heteroatom on the photobehaviour of 2,5-( $\mathrm{PhE})_{2} \mathrm{P}(2$, with linear conjugation) in non-polar solvent and in $30 / 70 \mathrm{CH}_{3} \mathrm{CN} / \mathrm{H}_{2} \mathrm{O}$ at $\mathrm{pH} 9$ and 2 .

\begin{tabular}{ccccc}
\hline $\begin{array}{c}\text { solvent } \\
\text { compound }\end{array}$ & MCH/3MP & \multicolumn{2}{c}{ aqueous medium } \\
\hline$\Phi_{\mathrm{F}}$ & $0.84-(\mathrm{PhE})_{2} \mathrm{~B}^{\mathrm{a})}$ & $2^{\mathrm{a})}$ & 0.32 & 0.14 \\
$\tau_{\mathrm{F}}(\mathrm{ns})$ & 1.7 & 0.29 & $0.57 / 1.1$ & $0.45 / 1.2$ \\
$\mathrm{k}_{\mathrm{F}}\left(10^{8} \mathrm{~s}^{-1}\right)$ & 4.3 & $0.43 / 0.92$ & $4 . \mathrm{H}^{\mathrm{b})}$ & $1.8^{\mathrm{b})}$ \\
$\Phi_{\mathrm{EE} \rightarrow \mathrm{ZE}}$ & $<0.001$ & $4.0^{\mathrm{b})}$ & 0.15 & 0.31 \\
\hline
\end{tabular}

a) From ref. [22].

b) derived using a mean $\tau_{\mathrm{F}}$ value obtained by a mono-exponential treatment of the fluorescence decay.

uncertain owing to the very small enthalpy differences between the crowded and elongated species of Table 3. Nevertheless, a more refined calculation based on an ab-initio method at 3-21G level for the geometry optimization, carried out only for compound 3, resulted in an inversion of the rotamers stability, in agreement with our previous findings in organic solvent [21].

\subsection{Photophysical and photochemical properties.}

Linear conjugation (1,4- and 2,5-derivatives). Tables 4 and 5 show the solvent and protonation effect on the relaxation properties of compounds 1 and 2 compared with the parent hydrocarbon, 1,4-(PhE $)_{2} \mathrm{~B}$. The prevalent relaxation pathway of the latter is the radiative one, accompanied by a very low isomerization yield, negligible $S_{1} \rightarrow T_{1}$ intersystem crossing (ISC) but non-negligible $S_{1} \rightarrow S_{0}$ internal conversion (IC) [22, 24]. The nitrogen effect in the conjugative position leads to a redistribution of the excitation energy which reduces the order of the isomerizable bonds lowering the torsional barrier and favouring the ZE production to detriment of the radiative deactivation. In fact, the fluorimetric parameters in non-polar solvent [22], reported for comparison, show a net decrease in both $\phi_{\mathrm{F}}$ and $\tau_{\mathrm{F}}$ for the aza-analogue. The radiative kinetic parameter, $\mathrm{k}_{\mathrm{F}}$, remains practically unchanged at values above $10^{8} \mathrm{~s}^{-1}$, typical of allowed transitions. The competition between radiative and reactive relaxations pointed to a twisting process in the singlet potential energy curve $[22,24]$. A diabatic mechanism, common to stilbene and many stilbene-like molecules, is assumed to be operative. It involves IC from the perpendicular configuration in $S_{1}\left({ }^{1}\right.$ perp $\left.*\right)$ to the ground state ${ }^{1}$ perp and relaxation to $\mathrm{E}$ and $\mathrm{Z}$ with a partitioning factor of $\sim 0.5$ [31].

For compound 1 (Table 4), the emission quantum yield in aqueous medium, lower than that in organic solvent, is still substantial in the basic solution but decreases markedly for the protonated species. The lifetime has a similar trend. The fluorescence decay is monoexponential within the limits of the experimental error. The drastic reduction in the radiative parameters is accompanied by an increase in the $\mathrm{EE} \rightarrow$ ZE photoisomerization which proceeds with a quantum yield slightly higher than that in organic solvent, at pH 9, and almost doubled in acid media. In fact, the fluorescence yield decreases by a factor of $\sim 50$, while the isomerization yield increases by a factor of more than 600 , with respect to the parent hydrocarbon, and reaches at $\mathrm{pH} 2$ a value close or higher than the maximum value (50\%) expected for a diabatic mechanism. The effect of protonation is then quite important to drive the relaxation of the excited molecule along the reactive pathway.

The photobehaviour of compound 2 (Table 5) is not much different from that of $\mathbf{1}$. Howerer, the decrease in $\phi_{\mathrm{F}}$ and the corresponding increase in $\phi_{\mathrm{EE} \rightarrow \mathrm{ZE}}$ are less marked and a contribution of IC is probably operative. The fluorescence decay is biexponential at both basic and acid pH's, in agreement with the $\lambda_{\text {exc }}$ effect on the spectra (section 3.2). For this asymmetric compound, only the ZE isomer (with the cis double bond in ortho position with respect to the heteroatom) was produced in both basic and acid media. A small production of the other stereoisomer (EZ) was found only by sensitization with a triplet donor [22]. 
Table 6. Effect of the nitrogen heteroatom on the photobehaviour of $2,6-(\mathrm{PhE})_{2} \mathrm{P}(3$, with crossed conjugation) in non-polar solvent and in $30 / 70 \mathrm{CH}_{3} \mathrm{CN} / \mathrm{H}_{2} \mathrm{O}$ at $\mathrm{pH} 9$ and 2 .

\begin{tabular}{ccccc}
\hline solvent & \multicolumn{2}{c}{$\mathrm{MCH} / 3 \mathrm{MP}$} & \multicolumn{2}{c}{ aqueous medium } \\
compound & $\left.1,3-(\mathrm{PhE})_{2} \mathrm{~B}^{\mathrm{a}}\right)$ & $3^{\mathrm{a})}$ & 0.26 & $3-\mathrm{H}^{+}$ \\
\hline$\Phi_{\mathrm{F}}$ & $0.28 / 0.88$ & $0.24 / 0.72$ & $1.2 / 3.1$ & 0.034 \\
$\tau_{\mathrm{F}}(\mathrm{ns})$ & $18 / 7.5$ & $1.5 / 3.5$ & 1.1 & $0.34 / 1.1$ \\
$\mathrm{k}_{\mathrm{F}}\left(10^{8} \mathrm{~s}^{-1}\right)$ & $0.16 / 1.2$ & $1.6 / 2.1$ & 0.10 & 0.47 \\
$\Phi_{\mathrm{EE} \rightarrow \mathrm{ZE}}$ & 0.038 & $0.14 / 0.075$ & & 0.72 \\
\hline
\end{tabular}

a) From ref. [21].

Table 7. Effect of the nitrogen heteroatom on the photobehaviour of $2,6-(\mathrm{PhBu})_{2} \mathrm{P}(4$, with crossed conjugation) in non-polar solvent and in $40 / 60 \mathrm{CH}_{3} \mathrm{CN} / \mathrm{H}_{2} \mathrm{O}$ at $\mathrm{pH} 9$ and 2 .

\begin{tabular}{ccccc}
\hline solvent & \multicolumn{2}{c}{$\mathrm{MCH} / 3 \mathrm{MP}$} & \multicolumn{2}{c}{ aqueous medium } \\
compound & $1,3-(\mathrm{PhBu})_{2} \mathrm{~B}^{\mathrm{a})}$ & $4^{\mathrm{a})}$ & 4 & 0.30 \\
\hline$\Phi_{\mathrm{F}}$ & $0.44 / 0.71$ & $0.18 / 0.88$ & 3.6 & 0.038 \\
$\tau_{\mathrm{F}}(\mathrm{ns})$ & $26 / 8.0$ & $1.4 / 2.8$ & 0.83 & 1.3 \\
$\mathrm{k}_{\mathrm{F}}\left(10^{8} \mathrm{~s}^{-1}\right)$ & $0.17 / 0.89$ & $1.3 / 3.1$ & 0.11 & 0.28 \\
$\Phi_{\text {EEEE } \rightarrow \text { EZEE }}$ & 0.076 & 0.031 & 0.05 & not observed \\
$\Phi_{\text {EEEE } \rightarrow \text { ZEEE }}$ & 0.068 & 0.017 & & \\
\hline
\end{tabular}

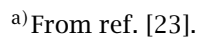

Crossed conjugation (1,3 and 2,6 derivatives). The radiative and reactive properties of compounds 3 and 4 in non-polar and protic solvents and at $\mathrm{pH} 2$ are shown in Tables 6 and 7 where they are compared with those of the parent hydrocarbons, 1,3-(PhE $)_{2} \mathrm{~B}$ and the corresponding butadienyl-derivative. Contrary to the 1,4 analogue, fluorescence remains the main deactivation pathway of these cross-conjugated distyrylbenzenes, at least for one rotamer. As observed in previous works from our [21] and other laboratories [24, 32, 33], the absorption spectrum of $1,3-(\mathrm{PhE})_{2} \mathrm{~B}$ is similar in position to that of $E$-stilbene (with almost double intensity) indicating that the cross-conjugation makes the two styryl chromophores practically independent. However, the emission spectrum is red-shifted and originates from a lower lying forbidden state of $\mathrm{A}_{\mathrm{g}}$ parentage, at least for one of the two observable conformers (see section 3.2). In compound 3, the effect of the heteroatom in the central ring does not affect much the fluorescence yield (Table 6) but modifies markedly the spectral properties and the conformational equilibrium (see later). Moreover, the yield of the reactive pathway increases in the aza-analogue, but less than for the linearly conjugated analogues. As suggested by NMR measurements, this behaviour is probably due to a type of intramolecular hydrogen bond interaction in one of the two rotamers, which keeps the molecule in a more rigid and planar conformation, thus reducing the IC efficiency [21]. This working hypothesis is confirmed by the behaviour in aqueous medium where the breaking of intramolecular $\mathrm{N} \cdots \mathrm{H}$ bonds restores the trend observed for the 1,4 aza-compound.
The emission quantum yield of 3 , lower than that in organic solvent, is still substantial in the basic solution and decreases further, by a factor of 7, at pH 2 . The lifetime, clearly biexponential, as found in organic solvent [21], is also quite smaller, by a factor of $\sim 3$, in acid medium. The decay was better investigated at different $\lambda_{\text {exc }}$ and $\lambda_{\text {em }}$ values (Table 8 ). On increasing $\lambda_{\text {exc }}$, the two lifetimes change only slightly, almost within the limits of the experimental uncertainty, but the weight of the longer component increases and tends towards $100 \%$ when exciting at the tail of the absorption spectrum. This confirms the presence of two main ground state rotamers (probably I and II in Table 3), as reported for the same molecules in organic solvent [21], and indicates that the bathochromic one is the longer lived rotamer. A complete fluorimetric analysis of the conformational equilibrium, based on kinetic and statistical methods [34], successfully carried out for similar compounds [35], was out of the scope of the present work. Therefore, the parameters in Table 6 refer to the rotamer mixture excited at the maximum of the first absorption band, corresponding to similar abundances of the two species. Given the relatively small difference between the lifetimes of the two rotamers, the radiative rate constant was calculated by using the lifetime measured with a monoexponential treatment. A similar behaviour was found for compound 2 , as shown in Table 8 . Only negligible deviations from mono-exponential decay were observed for compound 4 .

The radiative kinetic parameter of 3 reveals an allowed transition at $\mathrm{pH} 9$, but is rather lower at $\mathrm{pH} 2$, 
Table 8. $\lambda_{\mathrm{exc}}$ effect on the fluorescence decay of neutral and protonated species of linear-(2) and cross-conjugated (3) compounds in $30 / 70 \mathrm{v} / \mathrm{v} \mathrm{CH}_{3} \mathrm{CN} / \mathrm{H}_{2} \mathrm{O}$ at room temperature.

\begin{tabular}{|c|c|c|c|c|c|c|}
\hline compound & & $\lambda_{\text {exc }}(n m)$ & $\lambda_{\mathrm{em}}(\mathrm{nm})$ & $\tau_{\text {I }}(\mathrm{ns})$ & $\tau_{\text {II }}(\mathrm{ns})$ & $\%$ (II) \\
\hline \multirow{6}{*}{2} & \multirow{3}{*}{$\mathrm{pH}=2$} & 380 & 500 & 0.43 & 1.18 & 46 \\
\hline & & 380 & 420 & 0.45 & & 0 \\
\hline & & 425 & 500 & 0.51 & 1.18 & 75 \\
\hline & \multirow{3}{*}{$\mathrm{pH}=9$} & 350 & 425 & 0.55 & 1.10 & 43 \\
\hline & & 350 & 385 & 0.58 & 1.25 & 17 \\
\hline & & 395 & 425 & & 0.93 & 100 \\
\hline \multirow{6}{*}{3} & \multirow{3}{*}{$\mathrm{pH}=2$} & 372 & 460 & 0.30 & 1.11 & 49 \\
\hline & & 400 & 460 & 0.35 & 1.14 & 73 \\
\hline & & 410 & $>420^{\mathrm{a})}$ & 0.36 & 1.12 & 82 \\
\hline & \multirow{3}{*}{$\mathrm{pH}=9$} & 289 & 408 & 1.20 & 3.42 & 89 \\
\hline & & 335 & 408 & 1.22 & 3.15 & 77 \\
\hline & & 375 & 408 & 1.43 & 3.47 & 95 \\
\hline
\end{tabular}

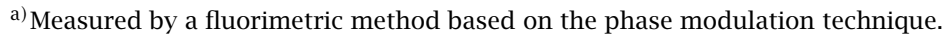

as found for the other compounds investigated and already observed for analogous compounds [20]. The $\mathrm{EE} \rightarrow \mathrm{ZE}$ isomerization yield of $\mathbf{3}$ is relatively small for the neutral molecule but, at pH 2, becomes higher (0.72) than the maximum value expected for a diabatic mechanism, as already reported for some styrylquinolines [36]. A tentative explanation of this behaviour could be related to a change in the shape of the $S_{0}$ and $S_{1}$ potential energy surfaces (different partitioning factor from the perpendicular configuration towards E and Z) caused by protonation.

The behaviour observed for the butadienyl derivative 4 (Table 7) partly reflects that of the ethenyl analogue. In the hydrocarbon and its aza-analogue in organic solvent, the photoreaction leads to two isomeric photoproducts, namely EZEE (rotation of the double bond adjacent to the central ring) and ZEEE (rotation of the double bond adjacent to the side ring). In this case, the introduction of the heteroatom in the central ring leads to a decrease in the isomerization yield in non-polar solvent, probably due to the intramolecular $\mathrm{N} \cdot \cdot \mathrm{H}$ interaction, which produces a more rigid and photostable structure. However, in protic solvent, a normal photoreactivity $[7,8,37]$ was again observed, with a prevalence of the twisting around the double bond adjacent to the pyridine ring; at $\mathrm{pH} 2$, EZEE becomes the unique photoproduct.

At $\mathrm{pH} \mathrm{2,} \mathrm{the} \mathrm{strong} \mathrm{decrease} \mathrm{in} \mathrm{the} \mathrm{fluorescence}$ yield is similar to the ethenyl derivative whereas the increase in reactivity is smaller since the longer chain favours non-radiative and non-reactive deactivation through the IC process. The lifetime of 4 offers a pattern of difficult interpretation owing to a larger number of possible conformers.

In the cross-conjugated compounds, an important difference between compounds having a central benzene or pyridine ring lies in the $\mathrm{k}_{\mathrm{F}}$ value of the longer- lived rotamer, which is markedly lower than that of the other rotamer, in the case of the hydrocarbons. This indicates that the emitting state of the longer-lived rotamer of 1,3-(PhE $)_{2} \mathrm{~B}$ and of the corresponding diene has a forbidden character. On the other hand, in compounds 3 and 4 , the observed reduction in the fluorescence lifetime of the neutral species is mainly related to the higher $\mathrm{k}_{\mathrm{F}}$ values, which indicate an allowed emitting state.

\section{CONCLUSIONS}

The position of the $\mathrm{N}$ atom in the side or central ring, the kind of conjugation and the $\mathrm{pH}$ of the solutions are important factors in determining the relaxation pathways of the excited state. The behaviour of the neutral molecules in the protic solvent was found similar to that in non-polar organic solvent, with a slight tendency to an increase in the yield of the reactive pathway to detriment of the radiative one. This behaviour is drastically emphasized in acid medium where the fluorescence quantum yield decreases by an order of magnitude for protonated compounds leading to high values of the photoreaction yield.

The presence of two main conformers, already observed in organic solvent for compound 2 [22] and for cross-conjugated compounds [21, 23], remains also in aqueous solution making the study more interesting but also more complicated.

The intermolecular interaction of these azacompounds with the protic solvent breaks the intramolecular $\mathrm{N}-\mathrm{H}$ interaction observed in non-protic solvent, particularly for the crossed compounds. Since this interaction has been reported to affect the conformational equilibrium (favouring the compressed rotamer) $[21,23,35]$ and to reduce the photoreactivity (which increases very little with respect to the 
hydrocarbon) [21-23], the protic solvent allows a substantial increase in the isomerization yield to be again observed.

An interesting effect was observed in the butadienyl derivative 4 where the basic species leads to two isomeric photoproducts whereas the acid medium, in addition to the general increase in the isomerization yield, selectively leads the EZEE isomer with the cis bond adjacent to the central pyridine ring.

The present results offer a picture of the different behaviour of the neutral and protonated species and indicate the experimental conditions suitable for a selective choice of a preferred relaxation pathway.

\section{ACKNOWLEDGMENTS}

This research was funded by the Ministero dell'Istruzione, dell'Università e della Ricerca (Rome) and the Perugia University in the framework of the Programmi di Ricerca di Interesse Nazionale (project: "Photoprocesses of interest for applications"). The authors thank Dr. Silvia Pannacci for her collaboration in the initial part of this research work and Mr. Danilo Pannacci for his technical assistance.

\section{REFERENCES}

[1] P. Bortolus, G. Cauzzo, U. Mazzucato, and G. Galiazzo, Z. Phys. Chem. N. F. 51 (1966), 264.

[2] G. Bartocci, U. Mazzucato, F. Masetti, and G. Galiazzo, J. Phys. Chem. 84 (1980), 847.

[3] U. Mazzucato, Pure Appl. Chem. 54 (1982), 1705 and references therein.

[4] F. Barigelletti, S. Dellonte, G. Orlandi, G. Bartocci, F. Masetti, and U. Mazzucato, J. Chem. Soc. Faraday Trans.1 80 (1984), 1123.

[5] G. Marconi, G. Bartocci, U. Mazzucato, A. Spalletti, F. Abbate, L. Angeloni, and E. Castellucci, Chem. Phys. 196 (1995), 383 and references therein.

[6] G. Marconi, G. Bartocci, U. Mazzucato, A. Spalletti, F. Abbate, L. Angeloni, and E. Castellucci, Am. Inst. Phys., Conf. Proc. 364 (1996), 175.

[7] G. Bartocci, G. Galiazzo, U. Mazzucato, and A. Spalletti, Phys. Chem. Chem. Phys. 3 (2001), 379.

[8] G. Bartocci, G. Galiazzo, L. Latterini, E. Marri, U. Mazzucato, and A. Spalletti, Phys. Chem. Chem. Phys. 4 (2002), 2911.

[9] D. G. Whitten and M. T. McCall, J. Am. Chem. Soc. 91 (1969), 5097.

[10] Y. J. Lee, D. G. Whitten, and L. Pedersen, J. Am. Chem. Soc. 93 (1971), 6330.

[11] D. G. Whitten and Y. J. Lee, J. Am. Chem. Soc. 94 (1972), 9142.

[12] S. C. Shim, M. S. Kim, K. T. Lee, B. M. Jeong, and B. H. Lee, J. Photochem. Photobiol. A: Chem. 65 (1992), 121 and references therein.

[13] E. J. Shin, E. Y. Bae, S. H. Kim, H. K. Kang, and
S. C. Shim, J. Photochem. Photobiol. A: Chem. 107 (1997), 137.

[14] G. Cauzzo, G. Galiazzo, U. Mazzucato, and N. Mongiat, Tetrahedron 22 (1966), 589.

[15] G. Favaro, U. Mazzucato, and F. Masetti, J. Phys. Chem. 77 (1973), 601.

[16] G. Bartocci, P. Bortolus, and U. Mazzucato, J. Phys. Chem. 77 (1973), 605.

[17] G. Favaro, F. Masetti, U. Mazzucato, and P. Bortolus, J. Phys. Chem. 79 (1975), 2785.

[18] A. Spalletti, U. Mazzucato, G. G. Aloisi, and D. Pannacci, Z. Phys. Chem. N.F. 138 (1983), 199.

[19] H. Görner, F. Elisei, and U. Mazzucato, J. Phys. Chem. 95 (1991), 4000.

[20] U. Mazzucato and A. Spalletti, Photochem. Photobiol. Sci. 2 (2003), 282.

[21] L. Giglio, U. Mazzucato, G. Musumarra, and A. Spalletti, Phys. Chem. Chem. Phys. 2 (2000), 4005.

[22] E. Marri, D. Pannacci, G. Galiazzo, A. Spalletti, and U. Mazzucato, J. Phys. Chem. A 107 (2003), 11231.

[23] E. Marri, G. Galiazzo, and A. Spalletti, Photochem. Photobiol. Sci. 3 (2004), 205.

[24] H. Meier, Angew. Chem. Int. Ed. Engl. 31 (1992), 1399.

[25] G. Bartocci, U. Mazzucato, and A. Spalletti, Rec. Trav. Chim. Pays-Bas 114 (1995), 459.

[26] A. Spalletti, G. Bartocci, F. Elisei, F. Masetti, and U. Mazzucato, Proc. Indian Acad. Sci. (Chem. Sci.) 10 (1998), 297.

[27] G. Bartocci, F. Masetti, U. Mazzucato, A. Spalletti, I. Baraldi, and F. Momicchioli, J. Phys. Chem. 91 (1987), 4733.

[28] P. Gajdek, R. S. Becker, F. Elisei, U. Mazzucato, and A. Spalletti, J. Photochem. Photobiol. A: Chem. 100 (1996), 57.

[29] E. F. G. Herington, Disc. Faraday Soc. 9 (1950), 26.

[30] I. Baraldi, A. Spalletti, and D. Vanossi, Spectrochim. Acta 59A (2003), 75.

[31] J. Saltiel and Y.-P. Sun, Photochromism: Molecules and Systems, (H. Dürr and H. Bouas-Laurent, Eds.), Elsevier, Amsterdam, 1990, 64 and references therein.

[32] D. Oelkrug, K. Rempfer, E. Prass, and H. Meier, Z. Naturforsch. 43a (1988), 583.

[33] Y. Ito, Y. Uozu, T. Dote, M. Ueda, and T. Matsuura, J. Am. Chem. Soc. 110 (1988) 189.

[34] G. Bartocci, A. Spalletti, and U. Mazzucato, in Conformational Analysis of Molecules in Excited States, (J. Waluk, Ed.), Wiley-VCH, New York, 2000, ch. 5 and references therein.

[35] A. Spalletti, G. Cruciani, and U. Mazzucato, J. Mol. Struct. 612 (2002), 339.

[36] G. Galiazzo, P. Bortolus, and G. Gennari, Gazz. Chim. It. 120 (1990), 581.

[37] G. Bartocci, G. Galiazzo, G. Gennari, E. Marri, U. Mazzucato, and A. Spalletti, Chem. Phys. 272 (2001), 213. 


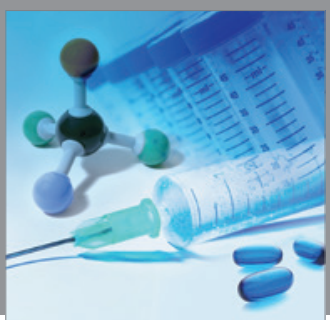

International Journal of

Medicinal Chemistry

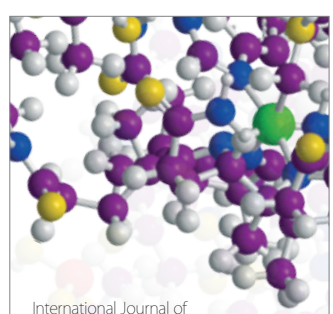

Carbohydrate Chemistry

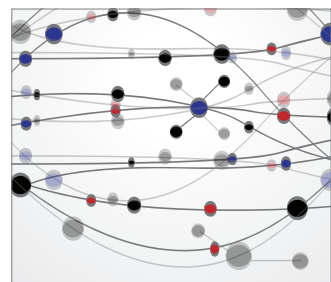

The Scientific World Journal
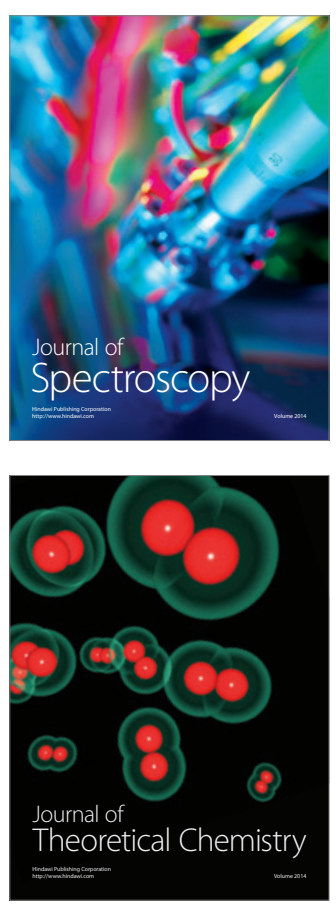
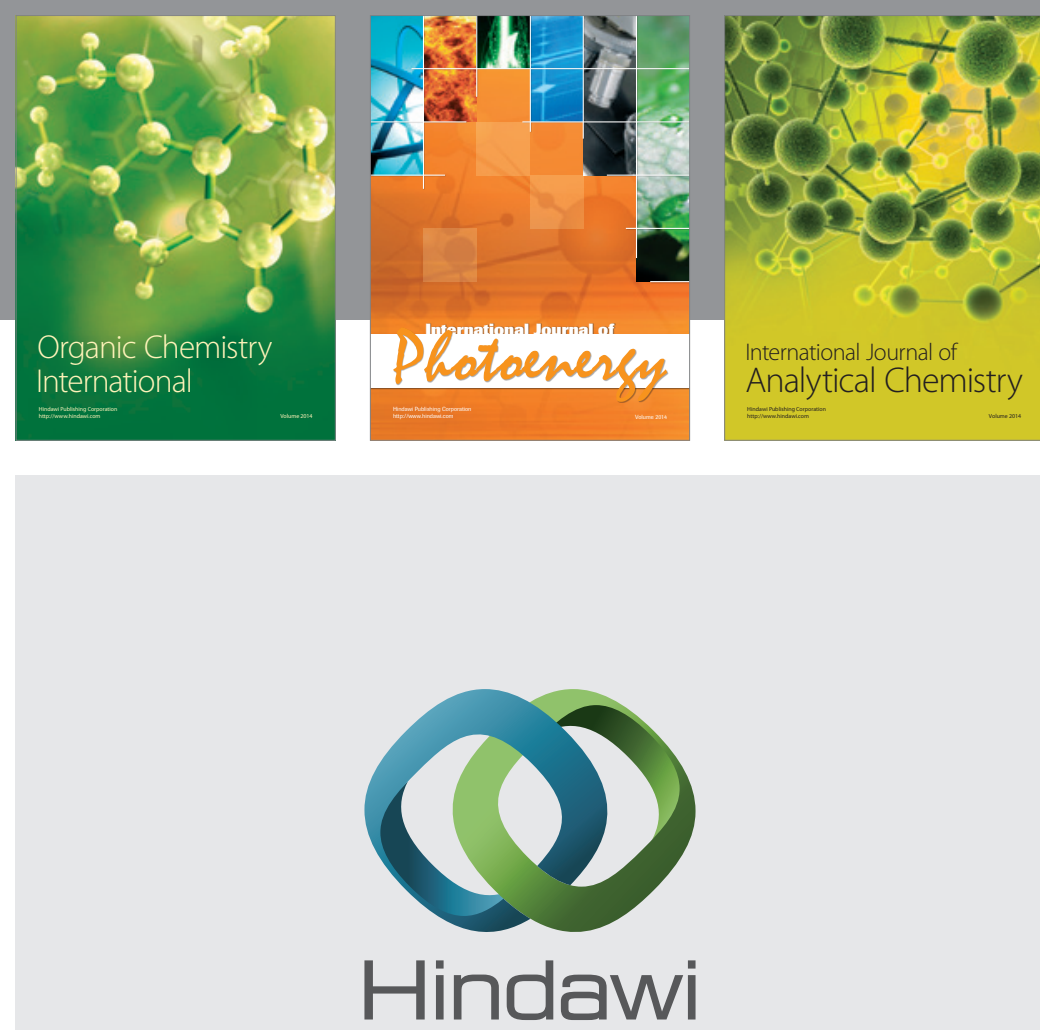

Submit your manuscripts at

http://www.hindawi.com
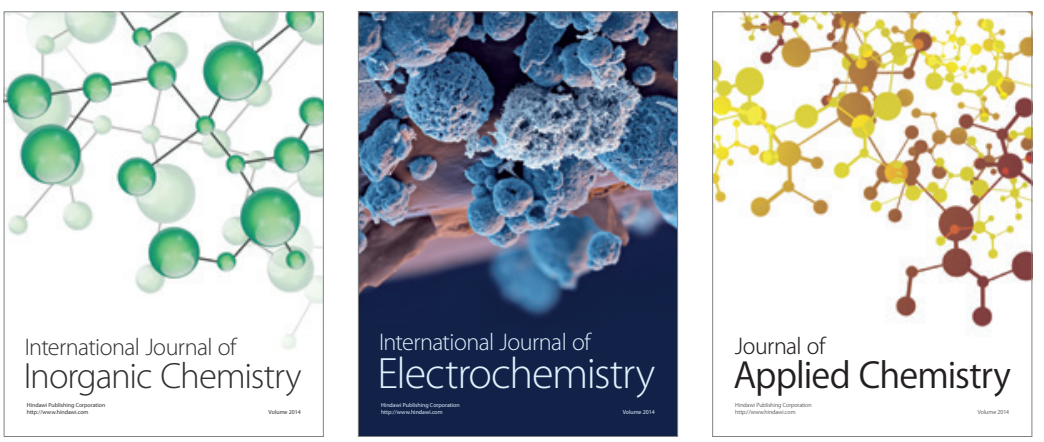

Journal of

Applied Chemistry
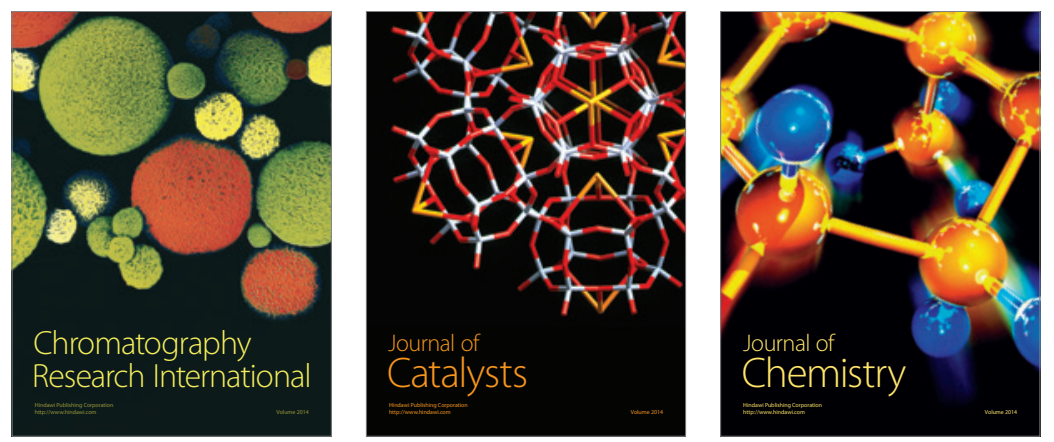
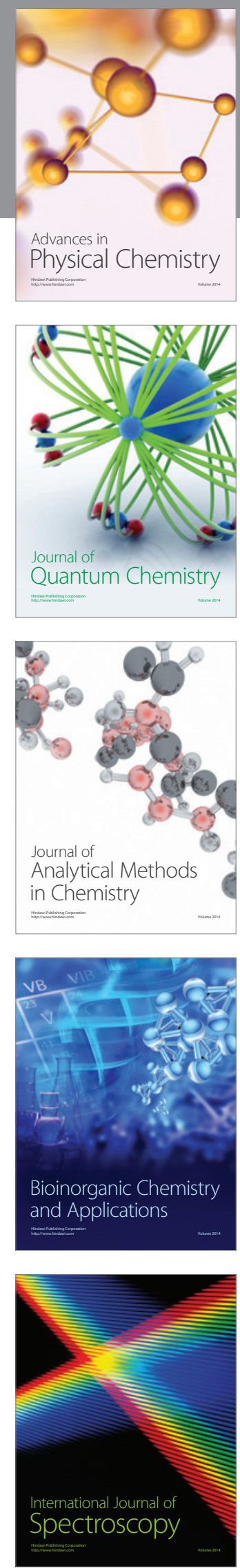